\title{
Investigation on Passive and Autonomous Mode Operation of Floating Gate Dosimeters
}

\author{
M. Brucoli ${ }^{\circledR}$, J. Cesari, S. Danzeca, M. Brugger, A. Masi, A. Pineda, L. Dusseau, and F. Wrobel ${ }^{\circledR}$
}

\begin{abstract}
In the context of achieving an efficient radiation monitoring system, while also aiming to increase the flexibility of the measurement system, the integrated digital and analog circuitries of the floating gate dosimeter (FGDOS) have been upgraded. The capability to control autonomously the recharge process and the easy access to the data allows exploiting the FGDOS in both the autonomous and passive mode. The former provides the cumulated dose measurement by a sensor coupled to a digital interface. The latter implies the reading of the dose only after the irradiation time, performing the dose detection by positioning the sensor without any supply. In this paper, the functionality and characterization of the new features of the FGDOS are demonstrated. The resolution, the dose range, and the error introduced on the measurements are discussed in detail by analyzing the results of irradiation experiments. The use of a filtering technique in different radiation environments is discussed, as well as the capability to retain the information after multiple readings and the power consumption for each configuration.
\end{abstract}

Index Terms-Dosimeter, floating gate dosimeter (FGDOS), ionizing radiation sensors, passive dosimeter, radiation monitoring.

\section{INTRODUCTION}

$\mathbf{T}$ HE monitoring of the ionizing radiation is a crucial task for a wide range of applications, which is often tackled by developing tailored radiation monitoring systems. In the framework of monitoring the radiation levels of large and distributed infrastructures, such as particle accelerators or nuclear power plants, the measurement systems are permanently installed to facilitate the comparison of the dose values in time and space. However, a flexible system that allows the repositioning of the dosimeters to investigate unexplored areas, with easy access to the data and avoiding the design and installation of a cabled network would provide a large benefit.

The need for noncabled radiation monitoring systems arises also when a highly accurate radiation field characterization is required, as it is the case for spallation sources or mixed

Manuscript received December 22, 2018; revised January 16, 2019 and January 18, 2019; accepted January 18, 2019. Date of publication February 1, 2019; date of current version July 16, 2019.

M. Brucoli is with the European Organization for Nuclear Research (CERN), CH-1211 Genève 23 Switzerland, and also with the Université de Montpellier, IES-UMR UM/CNRS 5214, F-34097 Montpellier, France (e-mail: matteo.brucoli@cern.ch).

J. Cesari and A. Pineda are with iC-Malaga, 07340 Alaró, Spain.

S. Danzeca, M. Brugger, and A. Masi are with the European Organization for Nuclear Research (CERN), CH-1211 Genève 23 Switzerland.

L. Dusseau and F. Wrobel are with the Université de Montpellier, IES-UMR UM/CNRS 5214, F-34097 Montpellier, France.

Color versions of one or more of the figures in this paper are available online at http://ieeexplore.ieee.org.

Digital Object Identifier 10.1109/TNS.2019.2895366 field radiation facilities. In particular, measurements on large surfaces or complex volumes require the mapping of the radiation field. In this context, the determination of the dose distribution on the device under test might not be straightforward, depending on the complexity of the device itself and the nonhomogeneity of the radiation field.

Aiming to improve the dosimetry resolution, several recent studies showed how silicon dosimeters based on floating gate structure can be good candidates for ionizing radiation monitoring (see [1]-[3]). In this concern, the floating gate dosimeter (FGDOS) developed by the company iC-Màlaga, named FGDOS, results a flexible sensor capable to measure dose levels as small as $0.5 \mathrm{mGy}$. Moreover, the suitability of the FGDOS as an ionizing radiation detector for mixed radiation field environments has been demonstrated in [4], where the dosimeter has been analyzed and compared with the RadFET.

With the purpose of providing a flexible monitoring system, this paper presents two different methods for measuring the dose by means of the FGDOS, hereafter called passive and autonomous mode. The passive mode permits to evaluate the dose by positioning the dosimeters where the radiation has to be measured: the output reading is performed before and after the irradiation and the sensors do not need any connection or supply during the radiation detection. As it will be shown, the dose range will be limited to the single discharge of the floating gate capacitor. To overcome this drawback, it is possible to use the sensor in Autonomous Mode, which allows the extension of the dose range up to the lifetime of the sensor. Thanks to the integration of the recharging circuitry on the FGDOS and the improvement of the communication controller, the online recording of the output signal becomes unnecessary, and the measurement can be performed by accessing occasionally the registers through the digital interface, which allows the communication with a microcontroller meant for reading the data and configuring the sensor.

In this way, it would be possible to develop flexible monitoring systems, for example, by implementing a network of detectors by reading each sensor through commercial wireless board capable of communicating by Serial Peripheral Interface (shortly SPI).

In this paper, the use of the FGDOS in passive and autonomous mode is discussed, following the preliminary results presented in [5]. The methods to calculate the dose are described, and different sources of error that affect the measurement are detailed. In addition to the characterization of the radiation response, several aspects of the operational modes 


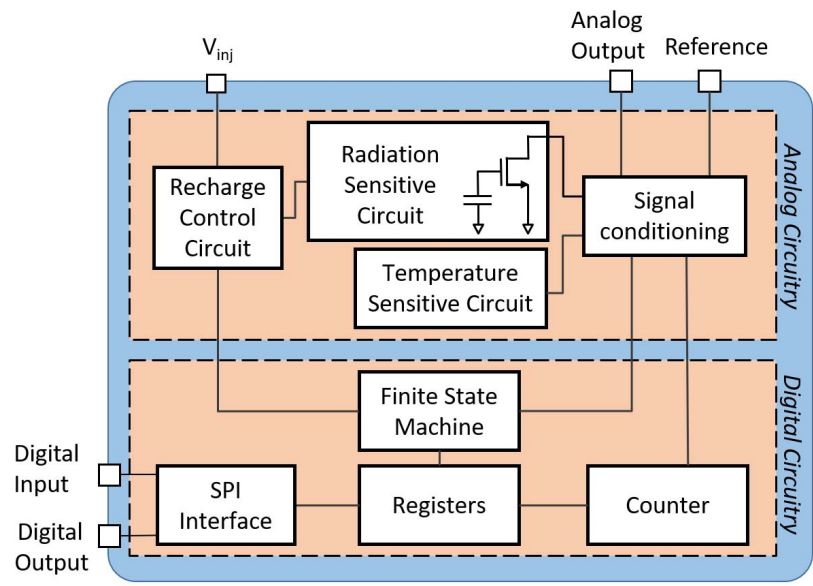

Fig. 1. Block diagram of the FGDOS architecture.

are investigated. The robustness against electrostatic discharge that might affect the measurements has been demonstrated. The effectiveness of moving average filters with respect to the dose rate variation is examined. The power consumption of the sensor is discussed with a view to develop limited power budget monitoring system (e.g., portable radiation monitoring, or for space applications).

\section{CONCEPT}

The FGDOS is a system on chip that can be represented by the architecture shown in Fig. 1. The radiation sensitive circuit is based on a single transistor whose gate is floating. The gate area is extended over the field oxide in order to increase the radiation sensitive volume (see [6, Fig. 1]). This structure can be modeled as a capacitor connected to the gate of a reading transistor. In order to measure the dose, the capacitor is positively precharged through an injector transistor [7]. The electron-hole pairs generated by the ionizing radiation in the field oxide are separated by the electric field: the holes are pushed toward the substrate, whereas the electrons are collected by the precharged floating gate. As a consequence, a portion of the charge stored in the capacitor is neutralized by the radiation-generated electrons, inducing a variation in the drain current of the transistor [8]. The signal conditioning circuit converts this current variation into a $5-\mathrm{V}$ square wave signal whose frequency is a function of the current variation, allowing the measurement of the absorbed dose [6], [9], [10]. This signal can be driven on loads such as PCB paths or 10-m coaxial cable. In this case, the frequency variation needs to be computed out of the chip, e.g., through an FPGA. Nevertheless, the FGDOS embeds a counter that evaluates the pulses of the square wave signal in a specific time window and writes the value into a dedicated register, providing a digital form of the frequency signal. The reading of the frequency can be performed by accessing the registers through SPI interface.

To obtain a linear radiation response, considering that the frequency versus dose characteristic is intrinsically nonlinear, the sensor is employed by exploiting a limited portion of the curve, which is named linear range. To keep the sensor working in this range, the floating gate capacitor must be recharged up to a target value whenever it is discharged below a certain threshold. The target and the threshold values limit the linear range in terms of both charge and output frequency. This procedure is implemented by the recharge control circuit, which compares the output frequency with the target and the threshold frequency to correctly enable and disable the recharge in order to force the sensor working in the linear range, whose limits can be set through the digital interface. A charge pump chain is integrated into the recharging circuit to provide the high voltage necessary to enable the tunneling current that crosses the gate oxide of the injector transistor. Moreover, it is possible to disable the internal recharge circuitry and implement an external recharge control by applying a high voltage through a dedicated pin to recharge the capacitor. If the recharge process is autonomously controlled by the FGDOS, the number of recharges is stored in an internal register to allow the calculation of the dose.

In addition, two configurations can be set to get different sensitivities, hereafter named high sensitivity (HS) and low sensitivity (LS) configuration. These sensitivities are obtained by switching between two reading transistors, whose dimensions determine different voltage-to-current gains.

Taking advantage of the capability of the FGDOS to retain the information both digitally (by storing the number of recharges) and physically (by storing a certain amount of charge in the floating gate capacitor), it is possible to exploit the sensor in passive and autonomous mode. The use of the sensor in passive mode allows the reading of the dose without requiring the sensor being biased or wired in any way. In this mode, the output frequency variation can be converted into dose in two different ways, which result in different dose ranges and resolutions, as it will be explained in Section III.

In the autonomous mode, the sensor is configured to control the recharge process and the absorbed dose can be easily calculated by reading the number of recharges and the output frequency at the reading time. In this way, the recording of the output data is not necessary, and the sensor needs only to be supplied.

\section{PASSIVE Mode}

The passive mode is implemented by evaluating the frequency before and after the irradiation: the frequency variation obtained can be converted into dose either by using the sensitivity of the FGDOS as a calibration factor or by exploiting the full characteristic as a calibration curve. The first method permits the calculation of the dose by linearly approximating its characteristic, whereas the second method requires the use of the whole calibration curve that needs to be stored and implemented through a lookup table.

In this paper, the dose is given in $\mathrm{Gy}(\mathrm{Si})$, although the unit reported is Gy for conciseness.

\section{A. Radiation Response Characterization}

The characterization of the radiation response is necessary to implement the passive mode measurement. For this reason, 


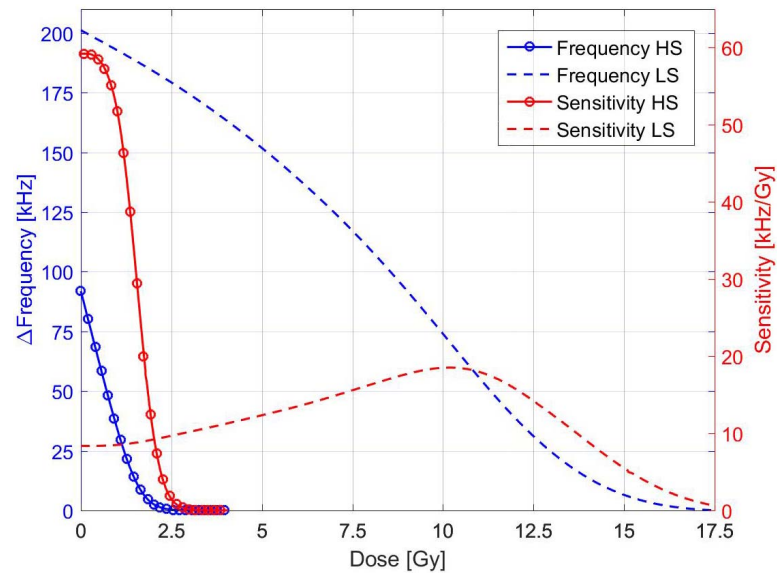

Fig. 2. Calibration curves (in blue) and sensitivity (in red) are plotted for the HS and LS configuration.

several irradiations were performed at the CC60 facility at CERN [11], where a gamma field is generated by a Co60 source. Here, the complete calibration curve has been achieved by irradiating the sensor configured in HS and LS. In Fig. 2, the calibration curve and the sensitivity (i.e., the derivative of the output frequency with respect to the dose) are plotted against the dose for both the configurations. As can be observed, the HS configuration, which is represented by the solid line, permits to obtain a much higher sensitivity than the LS configuration, although the measurable dose range is larger for the latter. The frequency interval corresponding to the linear range is $(50 / 80) \mathrm{kHz}$ and $(150 / 180) \mathrm{kHz}$ for the HS and LS configuration, respectively. The corresponding measurable dose range results in 0.51 and $2.72 \mathrm{~Gy}$.

\section{B. Linear Approximation}

The measurement of the dose through the linear approximation consists of the ratio between the frequency variation and the average of the sensitivity within the linear range, shortly $S_{\mathrm{LR}}$. The calibration of $S_{\mathrm{LR}}$ is also essential for the operation in autonomous mode, as it will be shown in Section IV. As long as the frequency variation stays within the linear portion of the characteristic, the error introduced will be negligible. In the case where the cumulated dose exceeds the linear range, the error introduced will strongly depend on the amount of dose exceeding the linear range, as can be observed in Fig. 3, where the error has been evaluated as the difference between the absorbed dose and the dose estimated by the linear approximation, as a function of the dose.

As it can be seen, the error increases abruptly as soon as the dose exceeds the linear range. However, if the measurement is not required being highly accurate, the dose range can be extended according to the error accepted. For instance, to get an error lower than $15 \%$, the dose range can be extended to 1.43 and $5.53 \mathrm{~Gy}$, respectively, for the HS and LS configuration. On the other hand, the maximum error given by the linear approximation within the linear range is 2.6 and $90 \mathrm{mGy}$ for the HS and LS configuration. In case, these ranges are not sufficient to cover the expected dose and the error constraints

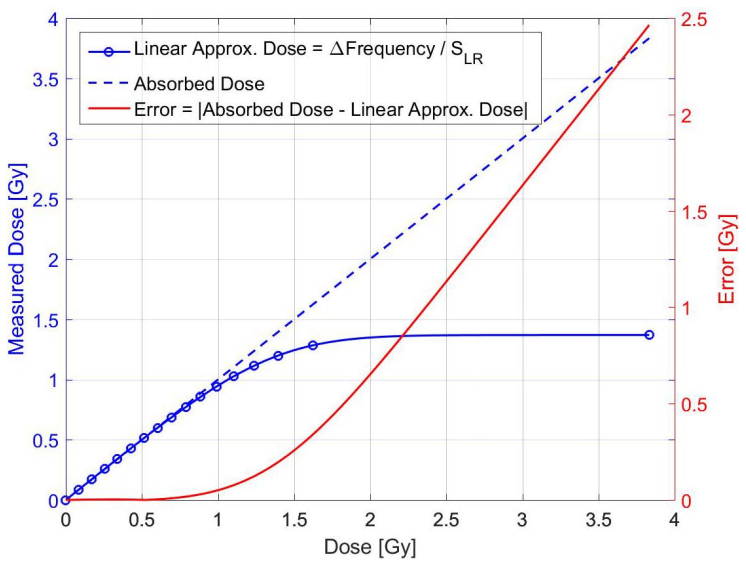

Fig. 3. Absolute error for the linear approximation (HS). The error (in red) is related to the right $y$-axis.

are more restrictive; it is convenient to evaluate the dose by considering the whole characteristic of the sensor.

\section{Calibration Curve}

The use of the whole characteristic as a calibration curve is more complicated, since a lookup table is required to convert the frequency variation into dose. On the contrary, a single value is needed by using the linear approximation, i.e., average sensitivity of the linear range $S_{\mathrm{LR}}$. However, this restriction is compensated by two important advantages: it allows the extension of the measurable dose range, and it does not introduce the error due to the approximation of the curve. The dose range extension can be evaluated by fixing the minimum measurable dose (namely, the dose resolution) allowed by the measurement. Since the sensitivity depends on the working point of the sensor, the dose resolution changes along the radiation response.

The dose resolution can be calculated as the ratio between the minimum detectable frequency and the sensitivity. As a consequence, the lower the sensitivity, the larger is the minimum detectable dose, i.e., the dose resolution. This implies that in the lower part of the characteristic, where the sensor loses most of its sensitivity, the minimum detectable dose increases. As the minimum detectable frequency resulted in $30 \mathrm{~Hz}$, the corresponding dose resolution has been evaluated and plotted against the dose in Fig. 4 for the HS configuration.

The resolution obtained in the linear range results in 0.5 and $3.1 \mathrm{mGy}$, respectively, for the HS and LS configuration. The span of the dose range is therefore limited by the resolution accepted. For example, considering acceptable a dose resolution 50\% higher than the one achieved in the linear range, the corresponding dose range is $1.18 \mathrm{~Gy}$ (HS configuration) and 12.25 Gy (LS configuration). In this way, it is possible to increase the dose range, respectively, to a factor 2.3 and 4.5 .

\section{Output Stability}

The stability of the output is a critical attribute to guarantee an accurate measurement in Passive Mode. To minimize 


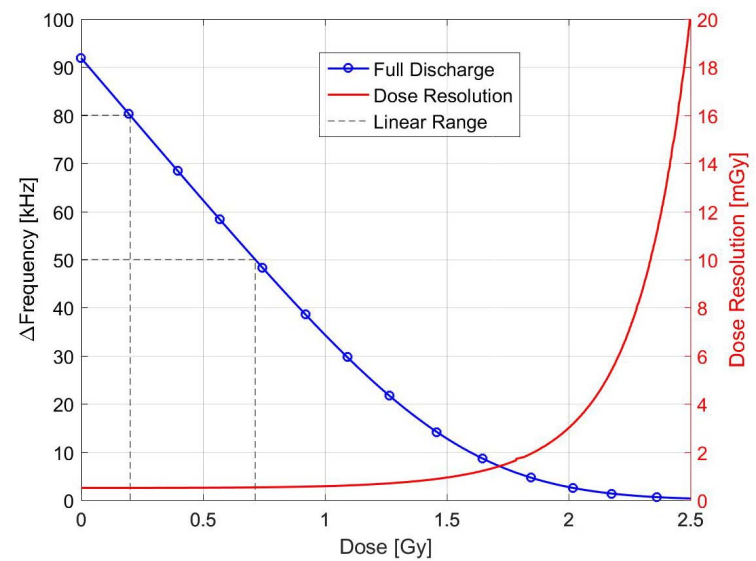

Fig. 4. Dose resolution considering a frequency noise of $30 \mathrm{~Hz}$ for the HS configuration.

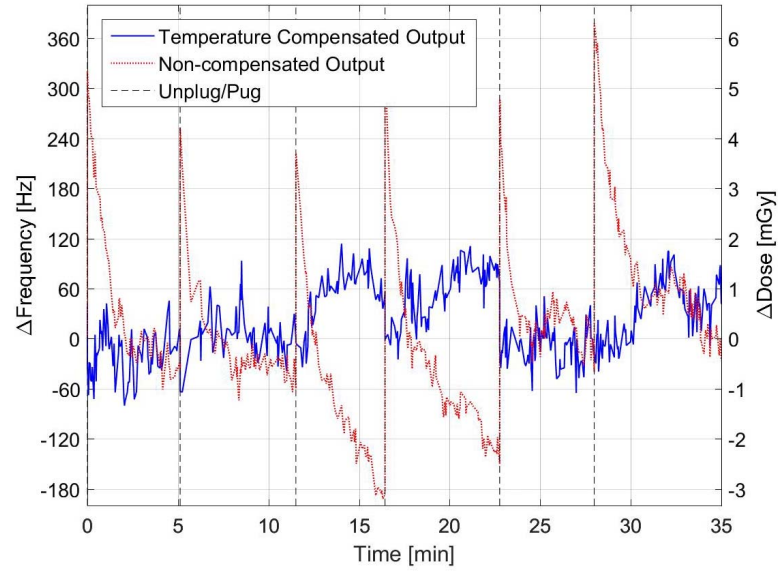

Fig. 5. Multiple readings of the output are shown. The vertical black lines indicate the removing of the sensor from the reading system. Between each reading, a period of $20 \mathrm{~min}$ was waited to bring the sensor to a stable temperature.

the error in the measurement, it is necessary to verify that during the pre- and post-irradiation reading of the dosimeter, the output is not affected by any unwished and accidental recharge or discharge of the floating gate capacitor due to possible electrostatic charges. Moreover, passive measurements can be strongly affected by temperature variations, as the readings are performed at a different time during which the temperature might change inducing frequency variations that must be compensated in order to improve the measurement accuracy. With this aim, the temperature compensation process presented in [4] has been applied.

In order to validate the stability of the output frequency, the sensor has been read and unplugged from the reading board repeatedly for one hundred times. Between each reading, the time necessary to cold down the chip was waited to have the same initial conditions. In Fig. 5, some of the readings are shown. On the right $y$-axis, the error due to the output fluctuation is reported for both the temperature compensated and the noncompensated signals. During the experiment, the maximum dose variation observed for the compensated and noncompensated signal results \pm 2 and $\pm 10 \mathrm{mGy}$, whereas
TABLE I

IRRADIATION TEST RESULTS

\begin{tabular}{cccc}
\hline \hline Configuration & Mode & Dose [Gy] & $\begin{array}{c}\text { Spread w.r.t } \\
\text { Active [\%] }\end{array}$ \\
\hline \multirow{2}{*}{ High } & Active & 0.227 & - \\
Sensitivity & Calibration Curve & 0.228 & 0.6 \\
& Linear Approx. & 0.230 & 1.6 \\
\hline \multirow{2}{*}{ Low } & Active & 0.849 & - \\
Sensitivity & Calibration Curve & 0.842 & 0.8 \\
& Linear Approx. & 0.768 & 9.5 \\
\hline
\end{tabular}

Table 1. Comparison of the Active and Passive Mode sensitivity obtained by irradiating different FGDOS in HS and LS configuration. The percentage difference is evaluated w.r.t. the Active Mode sensitivity and is indicated in the last column.

the standard deviation is 0.5 and $2 \mathrm{mGy}$, respectively. The larger variations on the noncompensated signal at each reading are due to the warming up of the chip during due to its functionality.

The multiple readings and the plug/unplug operation did not affect the charge stored in the capacitor, since the output signal did not decrease at the end of the experiment. As results, the passive mode procedure can be iterated as long as the sensor survives the radiation. Of course, the floating gate capacitor needs to be recharged every time that the dose exceeds the dose range to allow the measurement. Moreover, the temperature compensation is necessary to minimize the error in the dose measurement.

\section{E. Irradiation Experiments}

The passive mode has been tested by irradiating the FGDOS in both the HS and LS configuration with a dose rate ranging from 0.9 to $2.7 \mathrm{~Gy} / \mathrm{h}$. The dose was measured by means of an ionization chamber (PTW 23331), which was placed alongside the dosimeter. The distance from the source (more than $1 \mathrm{~m}$, depending on the dose rate) was enough to achieve high homogeneity of the radiation field to ensure a correct measurement of the dose. The sensors were precalibrated by reading the frequency variation throughout the irradiation (hereafter named active mode) and compared with the passive measurements in Table I. In the last column, the percentage difference between the active and passive mode with respect to the sensitivity measured in active mode is reported. As can be observed, the spread among the results is within $2 \%$, except for the linear approximation in LS configuration, which resulted much larger. This large difference is due to the nonoptimal choice of the linear range, which makes the sensor working in a rather extended portion of the characteristic, where the sensitivity changes consistently (see Fig. 2). In order to reduce this effect, the linear range should be shifted in a lower part of the curve, where the sensitivity variation is lower, such as for frequencies ranging from 50 to $80 \mathrm{kHz}$. For this purpose, the design of the temperature sensitive circuit must be modified in order to bias the temperature sensitive transistor in the same 
condition as the transistor that reads the capacitor. This would allow correctly compensating the temperature effect, which is necessary to perform the measurement in passive mode.

\section{Autonomous Mode}

The autonomous mode is a functionality that allows the reading of the dose by accessing the data through the digital interface, without the need of either recording the online measurement or externally controlling the recharge process. This function can be enabled by writing in the registers of the FGDOS through an SPI interface. The autonomous mode allows the calculation of the cumulated dose by the formula

$$
\text { Dose }=\frac{n r \times L R-\Delta \text { Freq }}{S_{\mathrm{LR}}}
$$

where $n r$ is the number of recharges, LR is the frequency span corresponding to the linear range (i.e., the difference between the target frequency and the threshold frequency), $\Delta$ Freq is the difference between the post-irradiation and the pre-irradiation frequency, and $S_{\mathrm{LR}}$ is the average sensitivity of the linear range. In order to control the recharge process, the logic of the ASIC reads and checks if the output frequency is below the threshold frequency. In this case, the logic triggers the recharge until the target frequency is reached. The number of recharges is stored in the registers to allow the computation of the dose. This technique permits the measurable dose range to be extended up to the lifetime of the sensor, which resulted in $300 \mathrm{~Gy}$.

\section{A. Recharge Time Error}

The dose calculated by (1) does not keep into account the finite time required for the recharge process to increase the frequency from the threshold to the target value. In fact, the formula approximates the recharge as an instantaneous event, although its duration is finite. The charge injection in the floating gate capacitor is triggered by applying a high voltage over the gate dioxide of a transistor, through the Fowler-Nordheim tunneling mechanism [12]. The high voltage is generated internally by the recharging circuitry, which includes a configurable charge pump chain. The time necessary to perform the recharge is hardly quantifiable because it depends on the dose rate. In fact, if the dose rate is too high, the charge generated by the radiation in the field oxide can neutralize a significant portion of the charge injected through the injector, consequently increasing the recharge time. Nevertheless, this phenomenon has been observed only for dose rates higher than $20 \mathrm{~Gy} / \mathrm{h}$. Assuming that the recharge time is independent of the dose rate, it is possible to estimate the relative error as the ratio of the recharge time over the time of a single discharge. This calculation overestimates the actual error because it does not keep into account the first and the last discharge, where no error due to the recharges is introduced. Therefore, since the time for a single discharge depends on the dose rate, the relative error can be expressed as a portion of the dose rate too. Since the recharge time has been measured for the HS and LS configuration in 10 and $65 \mathrm{~s}$, the multiplying factor to calculate the relative error results in $5.4 \times 10^{-3}$ and $6.6 \times 10^{-3}$, respectively. To get an idea on the influence of the error due to finite recharge time, a dose rate of $10 \mathrm{~Gy} / \mathrm{h}$ leads to a relative error of $5.4 \%$ and $6.6 \%$ for the HS and LS configuration. As a consequence, the dose calculated through (1) underestimates the actual absorbed dose depending on the dose rate. However, to reduce the error, it is worth making the recharge time as short as possible. For this reason, the number of stages involved in the charge pump chain has been made configurable, so that the injector voltage can be chosen among a set of voltage five values ranging between 17 and $19 \mathrm{~V}$. Nevertheless, if the highest value would not be enough, the charge pump can be disabled and the voltage can be applied through an external supply.

\section{B. Filtering}

The dose resolution can be improved by filtering a portion of the noise on the output signal by means of a low pass filter. Since the FGDOS output is a digital signal, a numeric filter must be implemented. The width of the passing band cannot be arbitrarily chosen, as the filter should not affect the signal variation induced by the radiation environment. Consequently, the filter needs to be designed according to the highest dose rate variation expected. Nevertheless, it is convenient to set a narrow bandwidth to cancel out as much noise power as possible. In this paper, a moving average filtering technique has been investigated. This technique consists in averaging the samples falling in a defined time window to produce a single sample.

To understand the influence of the filtering window width, two different experiments were performed at the CHARM facility at CERN, where a mixed radiation field is generated by a $24-\mathrm{GeV}$ proton beam impinging on a target [13]. During the experiments, the sensor was operating in autonomous mode and the output signal was measured online, converted into dose and filtered by using different moving windows, whose widths were set to 10,20 , and $40 \mathrm{~s}$.

The beam is extracted from the proton synchrotron accelerator, and it is delivered in bunches of $350 \mathrm{~ms}$, with a rate that changes depending on the beam availability. The number of bunches per minute delivered to the CHARM's target is normally within 1 and 6 . The nature of the beam makes the environment peculiar as the dose deposition due to the beamtarget interaction occurs within the bunch duration.

In the first experiment, the average dose rate generated by the beam-target interaction was about $250 \mathrm{mGy} / \mathrm{h}$ in the test location, whereas during the second experiment, the target was removed and the beam was dumped in such a way to obtain a much lower dose rate, which resulted on average $7.6 \mathrm{mGy} / \mathrm{h}$. In Fig. 6(a) and (b), the dose measured by the FGDOS is plotted for the first and the second experiment, respectively. As can be observed in Fig. 6(a), each step of the staircase shape shows the dose induced by the single bunch, which results in about $3 \mathrm{mGy}$ : this means that the maximum dose rate, which occurs within the bunch length, is $\sim 30 \mathrm{~Gy} / \mathrm{h}$. However, the filters smooth the signal and make the reading of the single bunch more difficult, as it is highlighted in the zoomed-in area in Fig. 6(a). Of course, the wider the window, the more intense is the smoothing of the signal. In the 


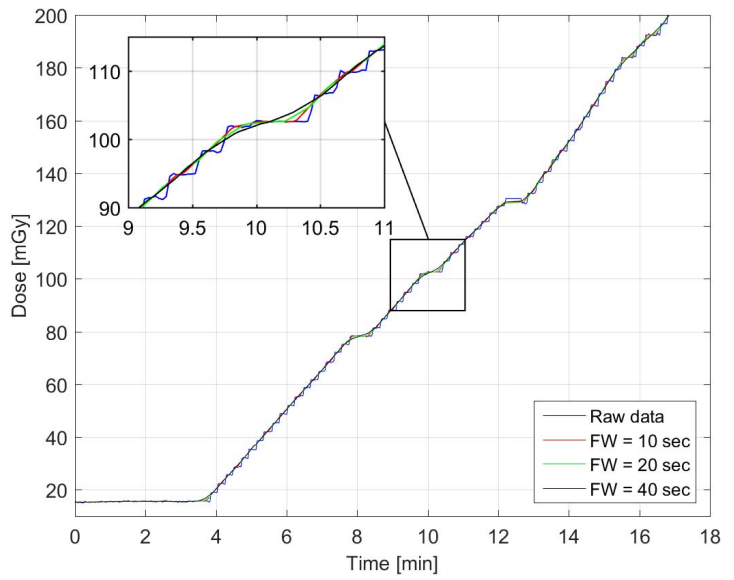

(a)

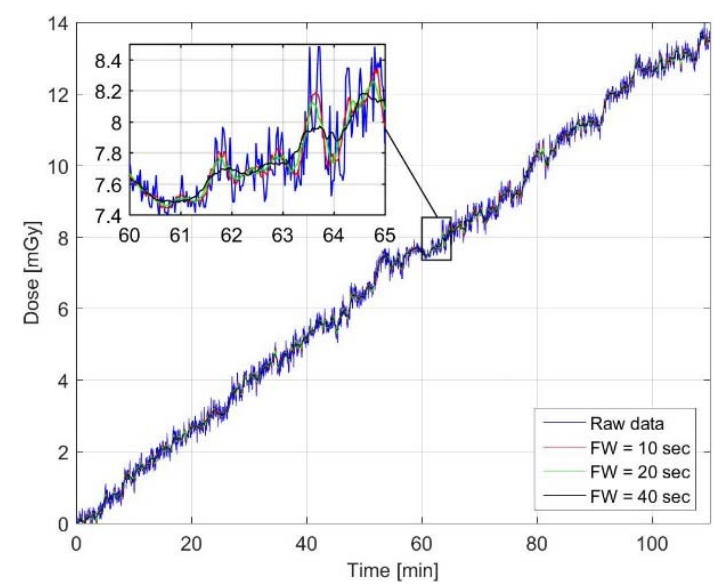

(b)

Fig. 6. Dose measured by the FGDOS in HS configuration at CHARM. The signal has been filtered by applying average windows with different widths $(10,20$, and $40 \mathrm{~s})$. The raw and the filtered signals are plotted for both the experiment. During (a) first experiment, the dose rate was $250 \mathrm{mGy} / \mathrm{h}$, whereas in (b) second experiment, the beam was dumped and the dose rate was 7.5 $\mathrm{mGy} / \mathrm{h}$.

second experiment, the dose delivered by the single bunch was not large enough to be distinguished by the sensor, as the test location was too far from the dump. In this case, the filtering significantly improves the dose reading: as can be observed in the highlighted zoomed in area box of Fig. 5(b), a large portion of the noise can be removed, although some slow fluctuations of the signal are only slightly attenuated even when applying wider filtering windows.

To explain this behavior, one can consider the normalized power noise spectrum plotted in Fig. 7, which shows a significant noise contribution at low frequency that cannot be canceled out with postprocessing filtering, as it would overlap the band of the signal. On Fig. 7, the magnitude of the frequency response of the moving average filter is plotted for different window widths.

In order to quantify the effectiveness of the filtering on the noise, the signal variation was evaluated in terms of standard deviation for different window widths while the sensor was placed in an oven to keep the temperature constant (see Table II).

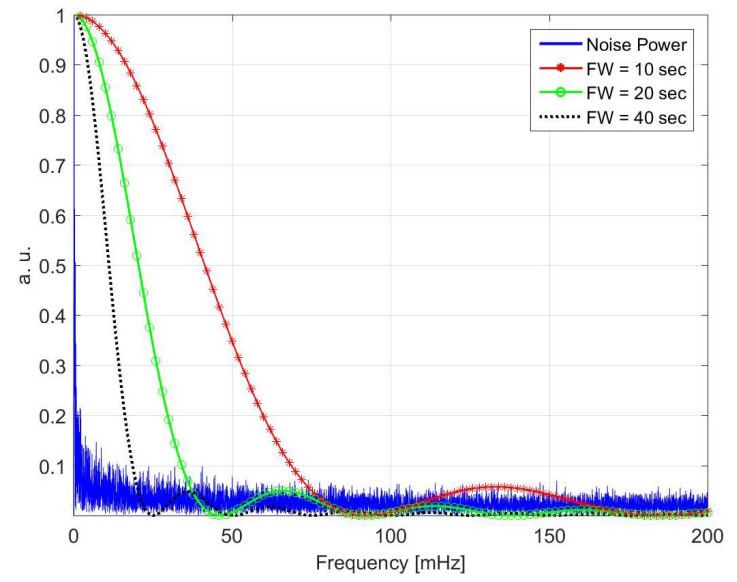

Fig. 7. Noise power spectrum and frequency response of the moving average filter for different window widths.

TABLE II

NOISE FILTERING

\begin{tabular}{ccc}
\hline \hline Width $[\mathrm{s}]$ & $\sigma[\mu \mathrm{Gy}]$ & $\Delta_{\text {REL }}[\%]$ \\
\hline Raw data & 538 & - \\
5 & 444 & 18 \\
10 & 419 & 22 \\
20 & 397 & 26 \\
40 & 379 & 30 \\
\hline
\end{tabular}

Table 2. Effect of the filtering on the noise amplitude. In the first column, the filter window width is reported, whereas $\sigma$ represents the standard deviation of the signal, and $\Delta_{\mathrm{REL}}$ represents the $\sigma$ variation in percentage with respect to the raw data.

This filtering technique can be implemented in both passive and autonomous modes. The passive mode does not require any restriction in terms of signal variation, as the sensor is read before and after the irradiation. For this reason, the filtering window width can be very long and the noise can be reduced according to Table II. On the other hand, the online reading of the output signal performed in autonomous mode during the first experiment permits to distinguish each single bunch delivered by the beam, which would not be observable when the filter is applied. Moreover, the filtering process must be disabled during the recharge process, as the signal variation is tens of times faster than the one induced by the dose. For this purpose, a bit of the register is set to 1 while the recharge in in progress. The filtering can be consequently enabled by the same bit.

\section{Sensitivity Degradation}

The sensitivity of dosimeters whose radiation-sensitive circuit is based on floating gate structure is affected by the dose, as the progressive accumulation of positive charge in the field oxide reduces the electric field, leading to a higher recombination rate and decreasing the carriers available for the neutralization of the charge stored in the capacitor [14]. To investigate the reliability of the sensor over its lifetime, the change of the sensitivity has been investigated by irradiating the sensor operating in autonomous mode with a dose 


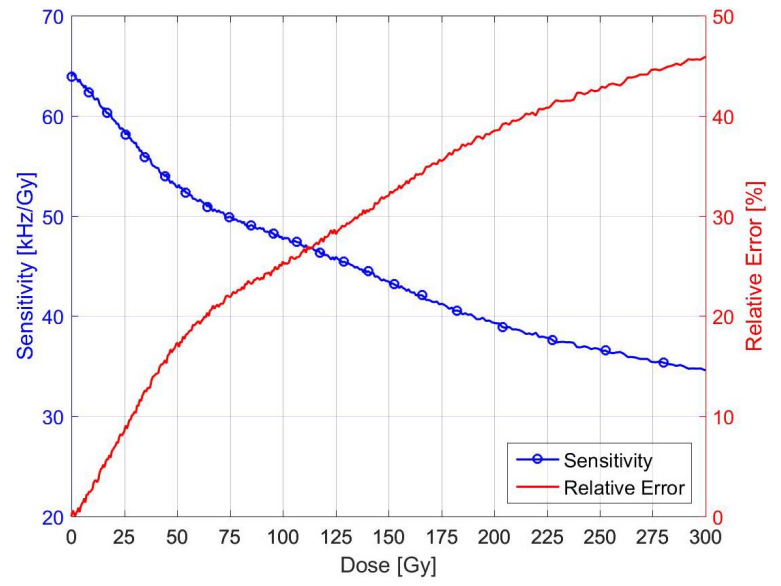

Fig. 8. Sensitivity variation over the dose is plotted for the HS configuration. On the right $y$-axis, the percentage variation of the sensitivity with respect to its initial value is reported

rate of $25 \mathrm{~Gy} / \mathrm{h}$. The sensitivity was measured for every discharge as the ratio between the frequency variation and the dose variation in the linear range. In Fig. 8 the sensitivity and percentage variation with respect to the initial value are plotted.

As can be observed, the sensitivity significantly decreases over the TID lifetime of the sensor. The highest degradation rate occurs in the first $50 \mathrm{~Gy}$, where the sensitivity changes with a rate of $0.34 \%$ per Gy. Afterward, it decreases with a rate of $0.14 \%$ per Gy between 50 and $200 \mathrm{~Gy}$ and it reaches $0.07 \%$ per Gy in the last 100 Gy. The higher sensitivity degradation rate corresponding to the lower doses might be due to the larger availability of empty traps that are gradually filled throughout the irradiation [15].

The calculation of the dose while the sensor works in autonomous mode made by (1) does not take into consideration that the sensitivity changes during the irradiation, introducing an error that must be considered. In fact, despite that the frequency range was forced by the logic of the sensor and the dose rate was constant, the dose interval between each recharge increased due to the degradation of the sensitivity. As a consequence, the dose calculated by (1) underestimates the dose actually absorbed. The percentage variation reported in Fig. 8 can be read as the additional absorbed dose that should be kept in to account for each discharge.

In Fig. 9, the absorbed dose and the dose calculated by (1) are compared, while the relative error with respect to the absorbed dose is reported on the right $y$-axis. It is important to notice that the relative error increases with the dose since it integrates the error made for every single discharge. Therefore, in order to drastically reduce the error, two different approaches can be used, depending on duration of the irradiation. For short irradiations, the sensor should be precalibrated to replace the sensitivity value $S_{\mathrm{LR}}$ in (1). Either for longer irradiation or for short irradiation when it is not possible to calibrate the sensor, the characterization reported in Fig. 8 has to be used to compensate the sensitivity degradation. For this purpose, a lookup table that takes the sensitivity degradation and the number of recharges as inputs can be easily implemented.

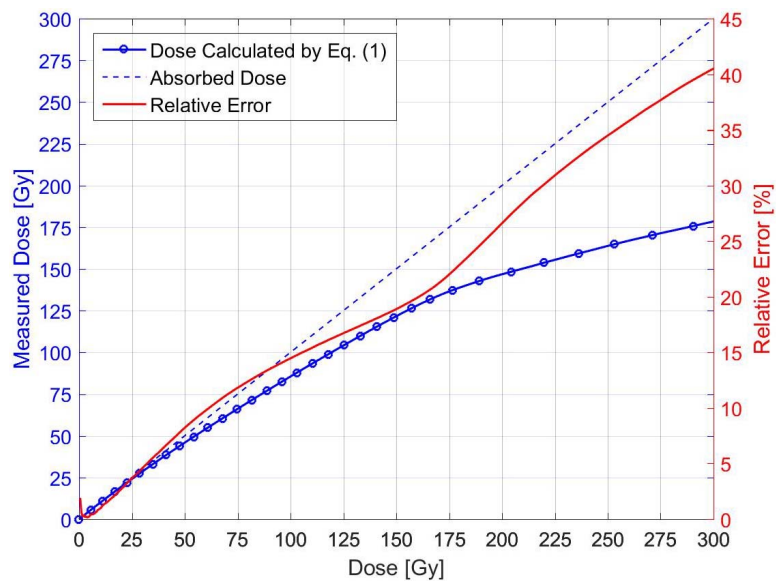

Fig. 9. Absorbed dose and the dose calculated by (2) are compared (left $y$-axis). The relative error with respect to the absorbed dose is reported on the right $y$-axis.

TABLE III

Power CONSUMPTION-Autonomous Mode

\begin{tabular}{cccc}
\hline \hline \multirow{2}{*}{ Sensitivity } & \multirow{2}{*}{ Output } & Recharge & Power [mW] \\
\hline \multirow{3}{*}{ High } & \multirow{2}{*}{ Buffer } & On & 20.47 \\
& & Off & 17.24 \\
& \multirow{2}{*}{ SPI } & On & 20.41 \\
& \multirow{2}{*}{ Low } & Off & 17.17 \\
& \multirow{2}{*}{ Buffer } & On & 10.60 \\
& \multirow{2}{*}{ SPI } & Off & 7.00 \\
& & On & 10.45 \\
\hline \hline
\end{tabular}

Table 3. Summary of the power consumption for different configurations of the sensor working in Autonomous Mode.

\section{Power Consumption}

In order to exploit the flexibility of this dosimeter and develop a portable radiation monitoring system, some consideration concerning the power consumption can be done to determine its operating life. Depending on the configuration used, the current required by the sensor changes significantly. For example, the HS configuration requires higher currents to increase the gain of the reading transistor, which significantly increase the power consumption. Again, the buffer that drives the $+5-\mathrm{V}$ square wave output signal can be disabled (if the output is read through the digital interface) to save some power. Moreover, the recharge circuitry increases the power consumption during the recharge process.

In Table III, a summary of the power consumption for different configurations is reported.

The energy budget can be estimated by considering the recharge time, which is constant for dose rates lower than $20 \mathrm{~Gy} / \mathrm{h}$, and the discharge time, which depends on the dose rate.

The capacity of a $5-\mathrm{V}$ battery that must supply the sensor over its whole TID lifetime ( $\sim 300 \mathrm{~Gy})$, considering a dose rate of $1 \mathrm{~Gy} / \mathrm{h}$, results 1.1 and $0.4 \mathrm{Ah}$, respectively, for the HS and LS configuration. Of course, the higher the dose rate, the shorter the lifetime (in unit time) of the sensor and the lower the energy budget required: the energy budget is inversely proportional to the dose rate. 
For applications with limited power budget (e.g., space applications), another strategy can be applied to minimize the power consumption. A monitoring system that supplies reads and, if necessary, recharges the sensor intermittently can be developed. In this way, the sensor operates repeatedly in passive mode, and therefore the dose between each reading can be calculated according with the characterization reported in Section III. A major constraint for such a system is that the reading must be performed before the capacitor is completely discharged. Therefore, since the output is not acquired continuously, the reading rate must be chosen according to the expected dose rate. For example, by reading the sensor configured in HS every $15 \mathrm{~min}$ and considering that each reading would last $\sim 20 \mathrm{~s}$, the energy budget required to supply the sensor over its lifetime is $12.3 \mathrm{mAh}$.

\section{CONCLUSION}

The functioning of the FGDOS in passive and autonomous modes have been demonstrated and characterized in this paper. A summary of the performance for each configuration is reported in Table IV. For the passive mode, two different methods have been shown. The error made by using the calibration curve resulted very low for both the configurations. The measurements made by the linear approximation resulted in good agreement with the absorbed dose when the sensor works in HS configuration. On the contrary, the LS configuration showed a significant mismatch between the measurements, due to the nonoptimal design of the linear range. To overcome this drawback, the biasing of the temperature sensitive circuit should be modified to correctly compensate the temperature effect, which is necessary to perform the measurement in passive mode. In addition, the retention of the output over multiple readings has been verified.

The functionality of the sensor working in autonomous mode has been presented. The analysis on the error introduced by the finite recharge time showed its dependence on the dose rate. To minimize this error, a faster recharge can be obtained by applying the high voltage externally. The use of the internal recharging circuitry requires the redesign of the charge pump chain. In addition, the ratio between the discharge and the recharge time can be further improved by increasing the linear range, i.e., reducing the dose rate dependence. However, the linear range extension is limited by the worsening of the resolution, which would make difficult the detection of threshold and target value performed by the embedded recharge process.

The use of the moving average filter has been tested through two experiments: the detection of large dose rate variations such as the one generated by the beam-to-target collisions at CHARM becomes difficult when the filter is applied. On the contrary, the noise can be significantly reduced for slow variations of the dose rate and for measurements in passive mode.

The degradation of the sensitivity has been characterized and the error induced by calculating the dose through (1) has been analyzed. Further studies should be performed to understand the suitability of the sensor for space applications,
TABLE IV

MODE COMPARISON

\begin{tabular}{ccccc}
\hline \hline \multirow{2}{*}{ Mode } & Method & Sensitivity & $\begin{array}{c}\text { Dose } \\
\text { Range [Gy] }\end{array}$ & Error \\
\hline \multirow{4}{*}{ Passive } & Linear & High & 0.51 & $2.6 \mathrm{mGy}$ \\
& Approx. & Low & 2.72 & $90 \mathrm{mGy}$ \\
\cline { 2 - 5 } & Calibration & High & 1.18 & $0.5 \mathrm{mGy}$ \\
& Curve & Low & 12.25 & $3.1 \mathrm{mGy}$ \\
\hline \multirow{2}{*}{ Autonomous } & \multirow{2}{*}{ Eq. (1) } & High & $\sim 300$ & $5.4 \cdot 10^{-3} \times \mathrm{DR} \%$ \\
& & Low & $\sim 300$ & $6.6 \cdot 10^{-3} \mathrm{x} \mathrm{DR} \%$ \\
\hline
\end{tabular}

Table 4. Summary of the characteristics of the operating modes. For the Autonomous Mode, the error depends on the dose rate (DR).

in particular investigating the possible dependence of the degradation mechanism on the temperature.

Finally, the power consumption of the sensor for each configuration has been discussed considering the use of the sensor for portable and limited power budget applications.

\section{REFERENCES}

[1] A. Faigon, I. M. Vazquez, S. Carbonetto, and M. García Inza, "Floating gate sensor for in-vivo dosimetry in radiation therapies. Design and first characterization," J. Phys., Conf. Ser., vol. 792, no. 1, 2017, Art. no. 012057.

[2] E. Pikhay, Y. Roizin, and Y. Nemirovsky, "Ultra-low power consuming direct radiation sensors based on floating gate structures," J. Low Power Electron. Appl., vol. 7, no. 3, p. 20, Jul. 2017.

[3] S. Danzeca et al., "Characterization and modeling of a floating gate dosimeter with gamma and protons at various energies," IEEE Trans. Nucl. Sci., vol. 61, no. 6, pp. 3451-3457, Dec. 2014.

[4] M. Brucoli et al., "Floating gate dosimeter suitability for accelerator-like environments," IEEE Trans. Nucl. Sci., vol. 64, no. 8, pp. 2054-2060, Aug. 2017.

[5] M. Brucoli et al., "Investigation on passive and autonomous mode operation of floating gate dosimeters," presented at the RADECS Conf., Gothenburg, Sweden, Sep. 2018.

[6] E. Garcia-Moreno et al., "Floating gate CMOS dosimeter with frequency output," IEEE Trans. Nucl. Sci., vol. 59, no. 2, pp. 373-378, Apr. 2012.

[7] A. Thomsen and M. A. Brooke, "A floating-gate MOSFET with tunneling injector fabricated using a standard double-polysilicon CMOS process," IEEE Electron Device Lett., vol. 12, no. 3, pp. 111-113, Mar. 1991.

[8] N. G. Tarr, G. F. Mackay, K. Shortt, and I. Thomson, "A floating gate MOSFET dosimeter requiring no external bias supply," IEEE Trans. Nucl. Sci., vol. 45, no. 3, pp. 1470-1474, Jun. 1998.

[9] E. G. Moreno, R. Picos, E. Isern, M. Roca, S. Bota, and K. Suenaga, "Radiation sensor compatible with standard CMOS technology," IEEE Trans. Nucl. Sci., vol. 56, no. 5, pp. 2910-2915, Oct. 2009.

[10] M. Álvarez, C. Hernando, J. Cesari, Á. Pineda, and E. García-Moreno, "Total ionizing dose characterization of a prototype floating gate MOSFET dosimeter for space applications," IEEE Trans. Nucl. Sci., vol. 60, no. 6, pp. 4281-4288, Dec. 2013.

[11] F. Pozzi et al., "CERN irradiation facilities," Radiat. Protection Dosimetry, vol. 180, nos. 1-4, pp. 120-124, Aug. 2018.

[12] J. A. Lopez-Villanueva, J. A. Jimenez-Tejada, P. Cartujo, J. Bausells, and J. E. Carceller, "Analysis of the effects of constant-current FowlerNordheim-tunneling injection with charge trapping inside the potential barrier," J. Appl. Phys., vol. 70, no. 7, pp. 3712-3720, Oct. 1991.

[13] J. Mekki, M. Brugger, R. G. Alia, A. Thornton, N. C. Dos Santos Mota, and S. Danzeca, "CHARM: A mixed field facility at CERN for radiation tests in ground, atmospheric, space and accelerator representative environments," IEEE Trans. Nucl. Sci., vol. 63, no. 4, pp. 2106-2114, Aug. 2016.

[14] T. R. Oldham and F. B. McLean, "Total ionizing dose effects in MOS oxides and devices," IEEE Trans. Nucl. Sci., vol. 50, no. 3, pp. 483-499, Jun. 2003.

[15] M. Brucoli et al., "Investigation on the sensitivity degradation of dosimeters based on floating gate structure," presented at the RADECS Conf., Geneva, Switzerland, Oct. 2017. 